Portland State University

PDXScholar

$11-1-2000$

\title{
Shubnikov-de Haas effect in the superconducting state of an organic superconductor
}

\author{
J. Wosnitza \\ S. Wanka \\ J. Hagel \\ R. Häussler \\ H. v. Löhneysen
}

See next page for additional authors

Follow this and additional works at: https://pdxscholar.library.pdx.edu/chem_fac

Part of the Physics Commons

Let us know how access to this document benefits you.

\section{Citation Details}

Wosnitza, J., Wanka, S., Hagel, J., Häussler, R., Löhneysen, H. V., Schlueter, J. A., Geiser, U., Nixon, P. G., Winter, R. W., Gard, G. L.(2000). Shubnikov-de Haas effect in the superconducting state of an organic superconductor. Physical Review B, 62, 11973

This Article is brought to you for free and open access. It has been accepted for inclusion in Chemistry Faculty Publications and Presentations by an authorized administrator of PDXScholar. Please contact us if we can make this document more accessible: pdxscholar@pdx.edu. 


\section{Authors}

J. Wosnitza, S. Wanka, J. Hagel, R. Häussler, H. v. Löhneysen, J. A. Schlueter, U. Geiser, P. G. Nixon, Rolf Walter Winter, and Gary L. Gard 


\title{
Shubnikov-de Haas effect in the superconducting state of an organic superconductor
}

\author{
J. Wosnitza, ${ }^{1}$ S. Wanka, ${ }^{1}$ J. Hagel, ${ }^{1}$ R. Häussler, ${ }^{1}$ H. v. Löhneysen, ${ }^{1}$ J. A. Schlueter, ${ }^{2}$ U. Geiser,${ }^{2}$ P. G. Nixon, ${ }^{3}$ R. W. Winter,${ }^{3}$ \\ and G. L. Gard ${ }^{3}$ \\ ${ }^{1}$ Physikalisches Institut, Universität Karlsruhe, D-76128 Karlsruhe, Germany \\ ${ }^{2}$ Chemistry and Materials Science Divisions, Argonne National Laboratory, Argonne, Illinois 60439 \\ ${ }^{3}$ Department of Chemistry, Portland State University, Portland, Oregon 97207
}

(Received 28 June 2000)

\begin{abstract}
We report the observation of Shubnikov-de Haas $(\mathrm{SdH})$ oscillations in the mixed state of the organic superconductor $\beta^{\prime \prime}$-(BEDT-TTF) ${ }_{2} \mathrm{SF}_{5} \mathrm{CH}_{2} \mathrm{CF}_{2} \mathrm{SO}_{3}\left(T_{c} \approx 4.4 \mathrm{~K}\right)$. At low temperatures the $\mathrm{SdH}$ oscillations persist clearly below the upper critical field $B_{c 2}(0) \approx 3.6 \mathrm{~T}$ almost down to the field where the resistivity vanishes. Rather unusually, no additional damping of the $\mathrm{SdH}$-signal amplitude — as well as of the de Haas-van Alphen amplitude - is observed in the superconducting state. This indicates that the damping in the vortex state of this quasi-two-dimensional superconductor is different in character to that observed for most three-dimensional materials.
\end{abstract}

After the report of de Haas-van Alphen (dHvA) oscillations in the superconducting state of $2 \mathrm{H}-\mathrm{NbSe}_{2}$ more than twenty years ago, ${ }^{1}$ this remarkable phenomenon has gained broad attention only recently. The dHvA effect in the vortex state seems to be a universal property as was confirmed experimentally for a large variety of different type-II superconductors. ${ }^{2}$ The observation of magnetic quantum oscillations, which usually is being taken as clear-cut evidence for the existence of a Fermi surface (FS), is highly unexpected in the mixed state of a superconductor where a spatially inhomogeneous energy gap evolves below the upper critical field $B_{c 2}$. Indeed, the microscopic mechanism leading to these oscillations is still lacking a satisfactory explanation. Much theoretical work has been devoted to an understanding of the commonly observed additional attenuation of the dHvA oscillations in the vortex state with respect to the normal-state damping. ${ }^{3-10}$ Most of these theories predict in addition to the usual exponential damping, caused by quasiparticle scattering with rate $\tau_{0}^{-1}$ due to static defects and impurities and described by the Dingle factor $R_{D}$ $=\exp \left(-r \pi m_{b} / e B \tau_{0}\right),{ }^{2,11}$ a further reduction factor $R_{S}$, which conveniently can be expressed in analogy to $R_{D}$ with an additional scattering rate $\tau_{s}^{-1}{ }^{2}$ Here, $r$ counts the harmonics of the oscillations, $m_{b}$ is the bare cyclotron effective mass unaffected by many-body interactions, and $\tau_{0}$ is related to the Dingle temperature via $T_{D}=\hbar / 2 \pi k_{B} \tau_{0}$. The predicted field dependence of the scattering rate $\tau_{s}^{-1}$ is governed by a possible spatial variation of the order parameter as calculated for various models. ${ }^{2}$ However, within the present models no consistent explanation of the experimental $\mathrm{dHvA}$ data for the different materials has been achieved.

To our knowledge, no Shubnikov-de Haas ( $\mathrm{SdH}$ ) oscillations have been reported so far for any material in the superconducting state. This seems to be of no surprise since the necessary ingredient for the observation of $\mathrm{SdH}$ oscillations is the presence of a finite resistivity which at first sight contradicts the principal property of a superconductor, namely $R=0$. However, below $B_{c 2}$ strongly type-II layered superconductors are characterized by a large reversible region where vortices are free to move and a finite resistivity exists. At lower fields or lower temperatures the strength for vortex pinning eventually exceeds the Lorentz force and $R$ indeed goes to zero. In most conventional metals the dHvA effect is experimentally easier to detect than $\mathrm{SdH}$ oscillations. The small $\mathrm{SdH}$ amplitude is caused by the typically very low relative number of electrons at the extremal area of the FS which contribute to magnetic quantum oscillations. This is fundamentally different for the quasi-two-dimensional (2D) organic metals with only one or two bands crossing the Fermi level $\epsilon_{F}$ and FS's which consist of only slightly corrugated cylinders. ${ }^{12}$ This means that although the total electron density $n$ is low (of the order of $10^{21} \mathrm{~cm}^{-3}$ ) almost all electrons at $\epsilon_{F}$ contribute to the oscillations. The amplitude of the $\mathrm{SdH}$ oscillations is, within a usually good approximation (see below), proportional to the relative change of the density of states $\Delta N\left(\epsilon_{F}\right) / N_{0}$ at the FS ( $N_{0}$ is the steady density of states). Therefore, for many $2 \mathrm{D}$ organic metals the $\mathrm{SdH}$ effect is easy to detect. ${ }^{12}$

The organic superconductor studied here is $\beta^{\prime \prime}$-(BEDT-TTF) ${ }_{2} \mathrm{SF}_{5} \mathrm{CH}_{2} \mathrm{CF}_{2} \mathrm{SO}_{3} \quad$ (BEDT-TTF is bisethylenedithio-tetrathiafulvalene) with a bulk $T_{c}=4.4 \mathrm{~K}$ and an upper critical field $B_{c 2} \approx 3.6 \mathrm{~T}$ for $T \rightarrow 0$ determined from the specific heat. ${ }^{13} \mathrm{SdH}$ (Ref. 14) as well as dHvA experiments ${ }^{15,16}$ show consistently one small 2D FS with an area of 5\% of the first 2D Brillouin zone. Band-structure calculations predict a somewhat larger $2 \mathrm{D}$ hole pocket in addition to a pair of wavy $1 \mathrm{D}$ electron sheets. ${ }^{14}$

The investigated crystals were grown by electrocrystallization. ${ }^{17}$ Here we present $\mathrm{SdH}$ and dHvA results for five selected crystals labeled $A-E$. Sample $A$ was measured in a ${ }^{3} \mathrm{He}$ cryostat down to $T=0.45 \mathrm{~K}$, sample $B$ in a toploading dilution refrigerator down to $25 \mathrm{mK}$. Thin current leads ( $15 \mu \mathrm{m}$ gold wire) were glued with graphite paste to the samples. The interplane resistance was measured with a four-point low-frequency ac-resistance bridge with a current of a few $\mu \mathrm{A}$. The magnetic field was oriented perpendicular to the BEDT-TTF planes along the $c$ axis, i.e., parallel to the current. This configuration minimizes the Hall contribution to the measured signal and allows a straightfor- 


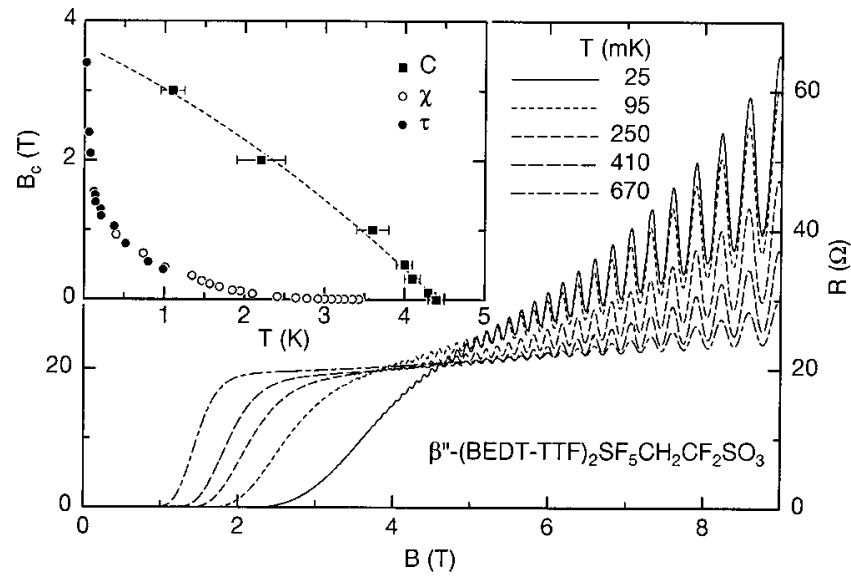

FIG. 1. Field dependence of the interplane resistance for selected temperatures (sample $B$ ). The inset shows the temperature dependence of the upper critical field as deduced by specific heat, $C$ and the irreversibility field as extracted from susceptibility, $\chi$ (Ref. 13 ), and torque, $\tau$, data. The dashed line is a quadratic fit through the $C$ data and extrapolates to $B_{c 2} \approx 3.6 \mathrm{~T}$.

ward extraction of the $\mathrm{SdH}$ signal, i.e., the relative conductance oscillations $\Delta \sigma=\sigma / \sigma_{b}-1$. Thereby the conductance $\sigma$ was calculated by inverting the measured resistance and the steady part of the conductance, $\sigma_{b}$, was fitted by a polynomial. The dHvA signals were measured with capacitance cantilever torque magnetometers. Sample $C$ was measured in a ${ }^{3} \mathrm{He}$ cryostat and the samples $D$ and $E$ in different dilution refrigerators. The magnetic field was oriented at about $14^{\circ}$ off the $c$ axis in order to increase the sensitivity of the torque magnetometer. At this orientation the $\mathrm{dHvA}$ frequency and $B_{c 2}$ is increased by only about $3 \%$.

Figure 1 shows the resistance of sample $B$ as a function of magnetic field for different temperatures. For $T=25 \mathrm{mK}$ and $T=95 \mathrm{mK}, \mathrm{SdH}$ oscillations are visible soon after a nonzero resistance is detected, depicted for $T=95 \mathrm{mK}$ more clearly in Fig. 2(a). The inset shows $\Delta \sigma$ between 1.95 and $2.5 \mathrm{~T}$ from which a $\mathrm{SdH}$ frequency of $F=199 \pm 1 \mathrm{~T}$ is derived. For higher fields the $\mathrm{SdH}$ amplitudes, residing on an approximately quadratically increasing background magnetoresistance, grow quickly. In agreement with previous results ${ }^{14-16}$ we observe one fundamental $\mathrm{SdH}$ frequency $F$ with an effective mass of $m_{c} \approx 2 m_{e}$, where $m_{e}$ is the free electron mass.

A central challenge for the present investigation is the reliable extraction of the upper critical field $B_{c 2}$. Around $B_{c 2}$ only feeble effects occur in $R$ and the magnetization. This, on the one side, hampers the exact determination of $B_{c 2}$, on the other hand it is the very existence of a large resistivity in the superconducting state which offers the possibility to observe $\mathrm{SdH}$ quantum oscillations in the superconducting state. One of the most reliable ways to determine $B_{c 2}$ are specificheat measurements which yield $B_{c 2}=3.6 \pm 0.5 \mathrm{~T},{ }^{13}$ although there are large error bars towards low temperatures. Much easier to detect is the irreversibility field, $B_{i r r}$, which is shown in the inset of Fig. 1. The data are extracted from ac-susceptibility measurements, $\chi$, (on a different crystal) ${ }^{13}$ and torque-magnetization data, $\tau$, as shown in Fig. 2(b). A clear hysteresis in $\tau$ is apparent between the up and down sweep with field. $B_{i r r}$ can be determined accurately from the field where the difference first deviates from zero [inset of

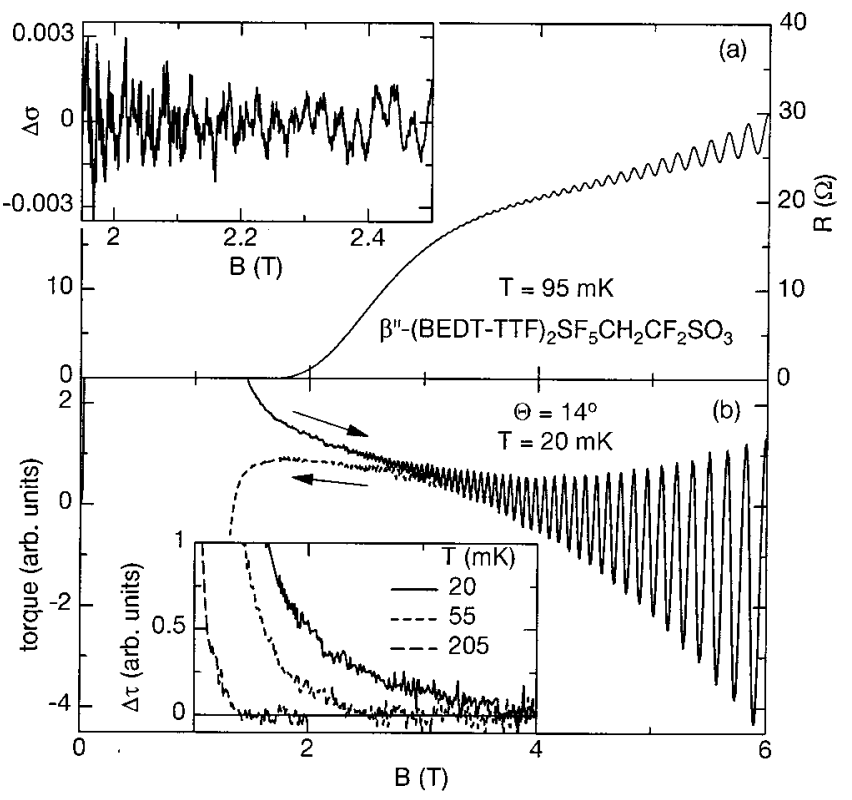

FIG. 2. (a) Field dependence of $R$ and the SdH signal (inset) of sample $B$ at $T=95 \mathrm{mK}$. (b) Torque signal of sample $D$ for up and down sweep. At about $3.4 \mathrm{~T}$ a clear hysteresis is visible. The inset shows the torque difference, $\Delta \tau$, for up and down sweeps at different temperatures. The irreversibility field is defined by the field where $\Delta \tau$ becomes zero.

Fig. 2(b)]. For $20 \mathrm{mK}$ this occurs at about $3.4 \mathrm{~T}$, which means that $B_{\mathrm{c} 2}$ must be definitely larger than this value giving support for the estimated $B_{\mathrm{c} 2}$ from specific-heat data. Towards higher temperatures, $B_{i r r}$ rapidly decreases, thereby increasing the region of the reversible Shubnikov phase. Below $B_{\text {irr }}$ clear dHvA oscillations are still visible [Fig. 2(b)] which can be well resolved down to about $2 \mathrm{~T}$ in a successive slower field sweep.

The fundamentally new phenomenon we observed is the detection of a $\mathrm{SdH}$ signal in the superconducting mixed state. At about $0.1 \mathrm{~K}$ the oscillations in sample $B$ are detectable down to very low fields (below $2 \mathrm{~T}$ ) sitting on a well resolvable background resistance $(\sim 0.7 \Omega$ at $1.95 \mathrm{~T})$ [Fig. 2(a)]. At $T=25 \mathrm{mK}$ the reversible field range is considerably reduced (see inset of Fig. 1) and, correspondingly, the $\mathrm{SdH}$ signal disappears already at $\sim 3 \mathrm{~T}$ in the background noise of the measurement. Figure 3 compares the $\mathrm{SdH}$ signals $\Delta \sigma$ of sample $A$ in the normal and superconducting states at $T$ $=0.45 \mathrm{~K}$ between 5 and $9 \mathrm{~T}$ and between 1.7 and $2 \mathrm{~T}$ (insets of Fig. 3). The fast Fourier transformation (FFT) of the data between 1.7 and $2 \mathrm{~T}$, i.e., well in the superconducting state, [Fig. 3(b)] reveals a clear peak at about $199 \mathrm{~T}$ at exactly the same frequency $F$ where the peak of the FFT in the normal state is located [Fig. 3(a)].

As mentioned, most theories for dHvA oscillations in the vortex state ${ }^{3-10}$ predict an additional attenuation of the oscillating signal with respect to the standard Lifshitz-Kosevich (LK) formula. ${ }^{11}$ For a detailed analysis of the field dependence of the dHvA and $\mathrm{SdH}$ oscillation amplitudes we performed FFT's over small field intervals (containing five to ten oscillations). The resulting FFT amplitudes $A_{F T}$ are shown in the so-called Dingle plot (Fig. 4), where $A_{F T} \sinh (X) T^{-1} B^{-1 / 2}$ is plotted on a logarithmic scale as a function of $1 / B$, with $X=2 \pi^{2} k_{B} m_{c} T / e \hbar B .{ }^{18}$ The straight 


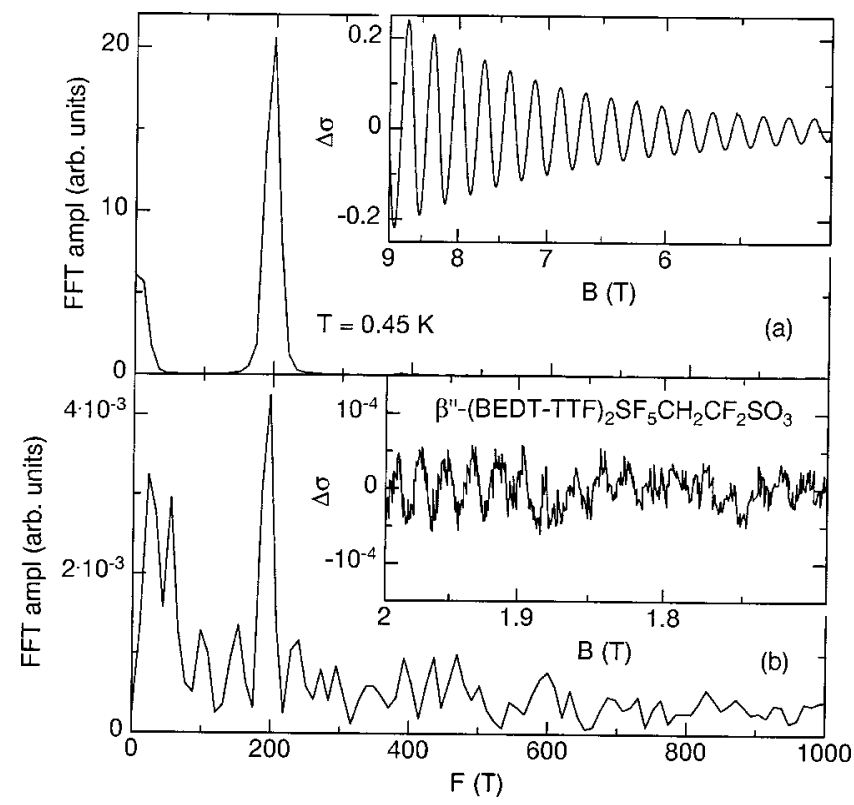

FIG. 3. Fourier spectra of the SdH signals (insets) of sample $A$ at $T=0.45 \mathrm{~K}$ in (a) the normal state $(5 \mathrm{~T} \leqslant B \leqslant 9 \mathrm{~T})$ and (b) in the superconducting state $(1.7 \mathrm{~T} \leqslant B \leqslant 2 \mathrm{~T})$. The $x$ axes of the insets are scaled linear in $1 / B$.

lines fitted to the data correspond to Dingle temperatures of $T_{D}=0.23 \mathrm{~K}$ for sample $A$ and $T_{D}=0.41 \pm 0.04 \mathrm{~K}$ for sample $B .{ }^{19}$ The latter value is not well defined since the data show a strong suppression of $A_{F T}$, due to a node in the $\mathrm{SdH}$ oscillations around $2.8 \mathrm{~T}$ which is presumably caused by mosaicity of the sample. Nevertheless, no additional damping of the $\mathrm{SdH}$ amplitude in the vortex state is realized for the present material.

We now compare this unusual $\mathrm{SdH}$ behavior with $\mathrm{dHvA}$ measurements. For different samples we were able to observe dHvA signals down to about $2.2 \mathrm{~T}$ at $T=0.41 \mathrm{~K}$ (sample $C$ ) and down to about $2 \mathrm{~T}$ at 20-30 $\mathrm{mK}$ (samples $D$ and $E$ ). Figure 4 shows the Dingle plot of the FFT data for

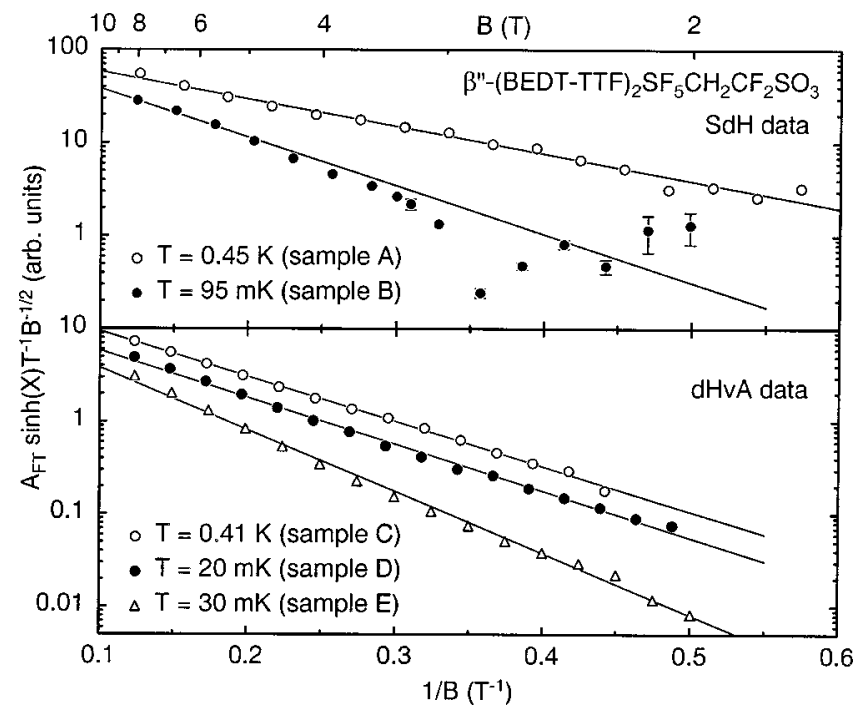

FIG. 4. Dingle plots of the $\mathrm{SdH}$ and dHvA amplitudes $A_{F T}$ of five different samples. The solid lines are fits to the data yielding $T_{\mathrm{D}}$ between $0.24 \mathrm{~K}$ (for sample $A$ ) and $0.52 \mathrm{~K}(E)$. all three samples. In line with the $\mathrm{SdH}$ data there is no additional attenuation visible below $B_{c 2}$ nor below $B_{i r r}$, i.e., below the opening of the hysteresis loop [Fig. 2(b)]. The solid lines represent the LK behavior with $T_{D}=0.38 \mathrm{~K}, T_{D}$ $=0.40 \mathrm{~K}$, and $T_{D}=0.52 \mathrm{~K}$ for sample $C, D$, and $E$, respectively. We omitted data to higher fields since above about 10 $\mathrm{T}$ the two-dimensionality leads to deviations from the 3D LK behavior. ${ }^{16}$ It is interesting to note that in previous $\mathrm{dHvA}$ experiments for the organic superconductor $\kappa$-(BEDT-TTF $)_{2} \mathrm{Cu}(\mathrm{NCS})_{2}$ as well, only a very gradual damping of the dHvA amplitude was observed around $B_{c 2} \cdot{ }^{20-22}$ Further on, no additional damping below $B_{c}$ was reported for $2 \mathrm{H}-\mathrm{NbSe}_{2}$ (Ref. 23) and $\mathrm{YNi}_{2} \mathrm{~B}_{2} \mathrm{C}{ }^{24}$

Our results show that there is no additional attenuation of the $\mathrm{SdH}$ and of the dHvA signal when going through and below $B_{c 2}$. When superconductivity sets in, an energy gap opens and the quasiparticle density of states at $\epsilon_{F}$ usually disappears. The reduced density and the gap at $\epsilon_{F}$, which may be anisotropic, is assumed ${ }^{3,4,6}$ to be the cause for the additional attenuation of the dHvA signal. The nonexistence of this attenuation in our $\mathrm{SdH}$ and $\mathrm{dHvA}$ signals may suggest that no gap at the Fermi surface exists. Although some experiments on organic superconductors give some hints for gap nodes, specific-heat data for $B=0$ clearly reveal an exponentially vanishing specific heat towards low temperatures, i.e., a fully gapped superconductor. ${ }^{13,25}$ This may be significantly different for $B>0$ where the quasiparticle spectrum is qualitatively altered by Landau quantization resulting in a new superconducting state with basically gapless quasiparticle excitations. ${ }^{2}$ This picture seems to be in line with the strongly reduced specific-heat jump in field and the absence of strong features in the magnetization.

While the dHvA effect, i.e., oscillations of the free-energy derivative $M=d F / d B$, presents an equilibrium property, the resistivity oscillations are an essentially different phenomenon including electron scattering in a magnetic field. The detailed theory is rather involved ${ }^{26}$ but the qualitative features can be rationalized by virtue of a simple argument. ${ }^{11}$ The probability for quasiparticle scattering is proportional to the number of states into which they can scatter. The density of states at the Fermi level oscillates with the field with an amplitude $\Delta N\left(\epsilon_{F}\right)$ and correspondingly the scattering probability. This finally leads to relative conductance oscillations $\Delta \sigma$, the amplitudes of which are proportional to $\Delta N\left(\epsilon_{F}\right) / N_{0}$.

The observation of a finite resistivity in the superconducting state is inherently coupled with moving vortices. On first sight, no Lorentz force acting on the (pancakelike) vortices is expected for the chosen parallel field-current configuration. However, due to the strong anisotropy of the organic material the charge carriers are believed to reside for a certain time within a highly conducting plane before a hoping process to the next plane occurs. In the Bardeen-Stephen model dissipation occurs by ordinary resistive processes in the vortex core. Other dissipative mechanisms by quasiparticle excitations can be described with the time-dependent Ginzburg-Landau wave function. ${ }^{27}$ The radius of a vortex core can be approximated by the coherence length $\xi_{\perp}$ $=\sqrt{\Phi_{0} / 2 \pi B_{c 2}} \approx 10 \mathrm{~nm}$, whereas the radius of a cyclotron orbit is $r=\hbar k_{F} / e B$ which is $\sim 260 \mathrm{~nm}$ at $2 \mathrm{~T}$. Thereby, $\Phi_{0}=h / 2 e$ is the flux quantum and a circular in-plane Fermi 
surface with wave vector $k_{F}=\sqrt{2 e F / \hbar}$ is assumed. This means that the observation of the SdH signal cannot simply be attributed to electrons on cyclotron orbits within a vortex core. Therefore, dissipative mechanisms within the superconducting regions must play the dominant role for the observed resistivity and the $\mathrm{SdH}$ oscillations. This dissipation originates from quasiparticle excitations as well as from the superfluid component. Both contributions may show an oscillating behavior with frequency as in the normal state which adds up to the $\mathrm{SdH}$ amplitude we observe. It is $a$ priori not clear how much each of these components contribute to the dHvA signal, i.e., to an oscillation in the density of states. For 2D, extremely type-II superconductors the calculated superconducting magnetization oscillation is predicted to be of the same order as the normal-electrons one. ${ }^{8}$ The sum of these contributions may add up to magnetic- oscillation signals which show no additional damping or even an increasing signal towards lower fields. ${ }^{8}$

In conclusion, we observed $\mathrm{SdH}$ oscillations in the mixed state of a strongly type-II 2D organic superconductor. No additional damping of the $\mathrm{SdH}$ as well as the dHvA signal below $B_{c 2}$ was observed. This suggests that in addition to the normal-electron contribution the superfluid component is responsible for the large signal. Our result shows that dissipative mechanisms in the superconducting region, i.e., outside of the vortex cores, are important for a finite resistivity in the superconducting state.

We thank P. Wölfle for stimulating discussions. The work at Karlsruhe was supported by the DFG. Work at Argonne National Laboratory was supported by the U.S. Dept. of Energy under Contract No. W-31-109-ENG-38. Work at Portland State University was supported by NSF Grant No. Che9904316 and the Petroleum Research Fund No. ACS-PRF 34624-AC7.
${ }^{1}$ J. E. Graebner and M. Robbins, Phys. Rev. Lett. 36, 422 (1976).

${ }^{2}$ T. J. B. M. Janssen et al., Phys. Rev. B 57, 11698 (1998), and references therein.

${ }^{3}$ K. Maki, Phys. Rev. B 44, 2861 (1991); M. J. Stephen, ibid. 45, 5481 (1992); A. Wasserman and M. Springford, Physica C 194196, 1801 (1994).

${ }^{4}$ K. Miyake, Physica B 186-188, 115 (1993).

${ }^{5}$ P. Miller and B. L. Györffy, J. Phys.: Condens. Matter 7, 5579 (1995).

${ }^{6}$ S. Dukan and Z. Tešanović, Phys. Rev. Lett. 74, 2311 (1995).

${ }^{7}$ M. R. Norman et al., Phys. Rev. B 51, 5927 (1995).

${ }^{8}$ T. Maniv et al., Phys. Rev. B 46, 8360 (1992); Physica C 235240, 1541 (1994); Solid State Commun. 101, 621 (1997).

${ }^{9}$ V. Zhuravlev et al., J. Phys.: Condens. Matter 11, L393 (1999).

${ }^{10}$ L. P. Gor'kov and J. R. Schrieffer, Phys. Rev. Lett. 80, 3360 (1998).

${ }^{11}$ D. Shoenberg, Magnetic Oscillations in Metals (Cambridge, University Press, Cambridge 1984).

${ }^{12}$ J. Wosnitza, Fermi Surfaces of Low-Dimensional Organic Metals and Superconductors (Springer, Berlin, 1996).

${ }^{13}$ S. Wanka et al., Phys. Rev. B 57, 3084 (1998); J. Wosnitza, Physica C 317-318, 98 (1999).
${ }^{14}$ D. Beckmann et al., Eur. Phys. J. B 1, 295 (1998).

${ }^{15}$ J. Wosnitza et al., Physica B 246-247, 104 (1998).

${ }^{16}$ J. Wosnitza et al., Phys. Rev. B 61, 7383 (2000).

${ }^{17}$ U. Geiser et al., J. Am. Chem. Soc. 118, 9996 (1996); J. A. Schlueter et al., Synth. Met. 85, 1453 (1997).

${ }^{18}$ For the $\mathrm{SdH}$ and the torque-dHvA amplitude the factor $B^{-1 / 2}$ occurs in the Dingle plot instead of $B^{1 / 2}$.

${ }^{19}$ For simplicity, we assumed $m_{b}=m_{c}$ for the determination of $T_{D}$, since the influence of many-body interactions are unknown. See also Ref. 2.

${ }^{20}$ P. J. van der Wel et al., Physica C 235-240, 2453 (1994).

${ }^{21}$ H. Ito et al., J. Supercond. 12, 525 (1999).

${ }^{22}$ T. Sasaki et al., Phys. Rev. B 57, 10889 (1998).

${ }^{23}$ E. Steep et al., Physica B 204, 162 (1995).

${ }^{24}$ M. Heinecke and K. Winzer, Z. Phys. B: Condens. Matter 98, 147 (1995).

${ }^{25}$ H. Elsinger et al., Phys. Rev. Lett. 84, 6098 (2000).

${ }^{26}$ E. N. Adams and T. D. Holstein, J. Phys. Chem. Solids 10, 254 (1959).

${ }^{27}$ M. Tinkham, Introduction to Superconductivity (McGraw-Hill, New York, 1996). 